\title{
Three-dimensional numerical analysis of terahertz radiation emitted from intrinsic Josephson junctions with hot spots
}

\author{
Hidehiro Asai, ${ }^{*}$ Masashi Tachiki, and Kazuo Kadowaki \\ Faculty of Pure and Applied Science, University of Tsukuba, Ten-noudai 1-1-1, Tsukuba, Ibaraki 305-8573, Japan
}

(Received 30 November 2011; revised manuscript received 25 January 2012; published 27 February 2012)

\begin{abstract}
In this study, we numerically investigate the terahertz radiation from mesa-structured intrinsic Josephson junctions (IJJs) using a three-dimensional calculation model. We assume an in-phase mode of the phase differences and calculate electromagnetic fields inside and outside of the IJJs simultaneously. We consider the appearance of a hot spot in the mesa where $j_{c}$ locally decreases and investigate the change of the radiation power with varying hot-spot positions. The radiation powers for three different hot-spot positions are calculated as functions of voltage. We observe strong radiation when the ac Josephson frequency satisfies the cavity resonance condition. Transverse-magnetic modes $\mathrm{TM}_{m, n}$ whose indices $m$ and $n$ are even appear regardless of the positions of hot spots. Meanwhile, the $\mathrm{TM}_{m, n}$ cavity modes whose $m$ or $n$ are odd appear only when the hot spots break the reflectional symmetry of the mesa structure. Moreover, we calculate the radiation patterns emitted by the IJJs at these cavity resonance conditions. The radiation patterns reflect the existence of two types of internal modes, that is, a uniform background mode and a cavity resonance mode.
\end{abstract}

DOI: 10.1103/PhysRevB.85.064521

PACS number(s): 74.50.+r, 74.25.Gz, 85.25.Cp

\section{INTRODUCTION}

Recently, the radiation of coherent terahertz waves from high- $T_{c}$ superconductor $\mathrm{Bi}_{2} \mathrm{Sr}_{2} \mathrm{CaCu}_{2} \mathrm{O}_{8+\delta}$ ( $\mathrm{Bi} 2212$ ) single crystal has attracted much attention. These samples generate $0.3-1-\mathrm{THz}$ waves and are expected to be possible candidates for a compact solid-state terahertz source. Since Bi2212 single crystals form intrinsic Josephson junctions (IJJs) that consist of the stacking of superconducting $\mathrm{CuO}_{2}$ layers and insulating Bi-Sr-O layers, ac Josephson currents flow through the crystals under dc bias voltages. Intense radiation has been reported in both experimental and theoretical studies at the voltages where the ac Josephson frequencies coincide with cavity resonant frequencies of the $\mathrm{Bi} 2212$ mesa. ${ }^{1-16}$ However, the precise nature of the radiation mechanism is not fully understood.

Klemm and Kadowaki have recently investigated the internal mode of the IJJs by analyzing the experimental radiation patterns $^{2-4}$ using antenna theory. ${ }^{17-19}$ For the interpretation of the experimental results, they assumed two types of radiation sources: the uniform part of ac Josephson current and the nonuniform part of ac Josephson current corresponding to cavity resonant modes. However, they did not calculate the phase dynamics in the IJJs. Meanwhile, Matsumoto and Koyama numerically investigated simultaneously the phase dynamics in the IJJs and radiation pattern using a two-dimensional model. ${ }^{13-15}$ They reported strong emissions from IJJs in the cavity resonance conditions and discussed the relation between the internal modes and the radiation patterns. However, in two-dimensional models, we cannot treat correctly the electromagnetic field emitted from threedimensional rectangular IJJs. Moreover, the radiation patterns emitted by inhomogeneous samples have not been investigated in previous studies. Recently, Wang et al. have reported the appearance of hot spots where the temperature is locally high, ${ }^{20-22}$ and these inhomogeneities are considered to affect the emission from the IJJs.

In this paper, we present a three-dimensional simulation of the radiation from IJJs to discuss the radiation properties of IJJs in further detail. In particular, we focus on the radiation properties of the IJJs having a hot spot. We consider the appearance of a hot spot in the mesa where $j_{c}$ locally decreases and investigate the change of the radiation power with the positions of the hot spots. The radiation power is calculated as a function of the voltage for three different hot-spot positions. We observe strong radiation when ac Josephson frequency satisfies the cavity resonance condition. Transverse-magnetic modes $\mathrm{TM}_{m, n}$ whose indices $m$ and $n$ are even appear regardless of the positions of hot spots. On the other hand, $\mathrm{TM}_{m, n}$ cavity modes whose $m$ or $n$ are odd appear only when the hot spots break the reflectional symmetry of the mesa structure. Moreover, the radiation patterns emitted from the IJJs reflect the coexistence of two types of internal modes, that is, the uniform background mode and the cavity resonance mode.

\section{CALCUlation METHOD}

We consider a rectangular mesa sample whose IJJs stack along the $z$ axis. The mesa is sandwiched by an infinite-size substrate and an upper electrode whose geometry is the same as the mesa. Figure 1 shows the schematic figure of our threedimensional calculation model. We assume that the substrate and the electrode are perfect electric conductors. The uniform external current parallel to the $z$ axis is injected from the upper electrode. For the outer boundary of the calculation region, we use the perfectly matched layer absorbing boundary condition. The dimensions of the mesas are as follows: width $w=0.48 \lambda_{c}$, length $l=0.72 \lambda_{c}$, and height $h=0.02 \lambda_{c}$, where $\lambda_{c}$ is the magnetic penetration depth along the IJJ plane. If we take $\lambda_{c}=100 \mu \mathrm{m}$, the sizes of the mesas become similar to the experimental studies.

In this study, we focus on the reversible type of emission observed at the reversible region of the $I-V$ curve. ${ }^{2,5,8,21}$ The thickness of the superconducting $\mathrm{CuO}_{2}$ layer of the $\mathrm{Bi} 2212$ single crystal is an atomic scale $(\sim 3 \AA)$ and comparable to a charge screening length. Hence, the phase differences between the IJJs interact with each other through the electric fields, which perpendicularly penetrate the junctions, and the phase 


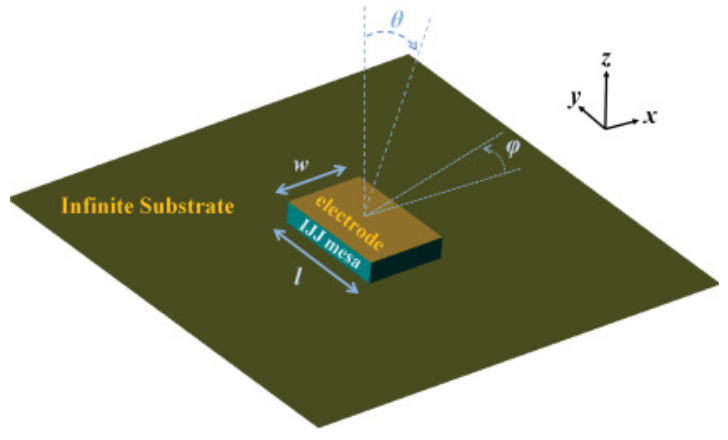

FIG. 1. (Color online) A schematic view of the three-dimensional calculation model.

synchronization will occur to keep the charge neutrality of the junctions. We assume the in-phase motion of the phase differences: all phase differences are equal to each other. ${ }^{11,13,14}$ In this assumption, the time evolution of the dimensionless electromagnetic fields and the phase differences in the IJJs are described by the following equations: ${ }^{11,13,14}$

$$
\begin{gathered}
\frac{\partial}{\partial t^{\prime}} P=E_{z}^{\prime}, \quad B_{x}^{\prime}=-\frac{\partial}{\partial y^{\prime}} P, \quad B_{y}^{\prime}=\frac{\partial}{\partial x^{\prime}} P, \\
\frac{\partial}{\partial t^{\prime}} E_{z}^{\prime}=\frac{1}{\epsilon_{c}}\left(\frac{\partial}{\partial x^{\prime}} B_{y}^{\prime}-\frac{\partial}{\partial y^{\prime}} B_{x}^{\prime}\right)+j_{\mathrm{ext}}^{\prime} \\
-j_{c}^{\prime}(x, y) \sin P-\beta E_{z}^{\prime},
\end{gathered}
$$

where $P$ is the phase difference between the IJJs, $E_{z}^{\prime}$ is the electric field, $B_{x}^{\prime}$ and $B_{y}^{\prime}$ are oscillation parts of the magnetic field, $j_{c}^{\prime}$ is the critical current, and $j_{\mathrm{ext}}^{\prime}$ is the homogeneous external current injected into the IJJs. The parameter $\beta=$ $4 \pi \lambda_{c} \sigma_{c} / c \sqrt{\epsilon_{c}}$ is the normalized conductivity, and $\epsilon_{c}$ is the dielectric constant of the junctions along the $c$ axis. In this study, we take $\epsilon_{c}=16$ and $\beta=0.075$. The value of $\beta$ is comparable to that of the normal conductivity of Bi2212 mesa under a large applied current. The $I$ - $V$ curves of the Bi2212 mesa show negative resistance in a large current region due to the joule heating, and the reversible type of emission has been reported in this region. ${ }^{2,5,8,21}$ Here we assume that the electric field parallel to the IJJ plane is negligibly small $\left(E_{x}=E_{y}=0\right)$ and the applied magnetic field is zero. In the above equations, we use dimensionless quantities as follows: length $x^{\prime}=x / \sqrt{\epsilon_{c}} \lambda_{c}$, time $t^{\prime}=\omega_{p} t$, where $\omega_{p}=c / \sqrt{\epsilon_{c}} \lambda_{c}$, electromagnetic field $E^{\prime}=\left(2 e d / \hbar \omega_{p}\right) E, B^{\prime}=\left(2 e d / \hbar \omega_{p}\right) B$, where $d$ is the thickness of the insulating layers of IJJs, and current $j^{\prime}=\left(8 \pi^{2} d \lambda_{c}^{2} / c \phi\right) j$. As described in Eqs. (1) and (2), the phase dynamics of the in-phase IJJs is similar to that in a single junction. However, the distances between the adjacent IJJ layers are extremely small; thus a large number of junctions contribute to the phase dynamics even in thin mesas whose thicknesses are approximately microns. This situation makes it possible for IJJs to emit a high-power terahertz wave.

In this study, we use a finite-difference time-domain method for calculating the electromagnetic field inside and outside of the mesa. In the region inside of the mesa, we set $E_{x}=E_{y}=$ $B_{z}=0$ and solve Eqs. (1) and (2). Meanwhile, in the region outside of the mesa, we solve three-dimensional Maxwell's equations in free space. The electromagnetic fields in both the regions are directly connected to each other at the side edges of
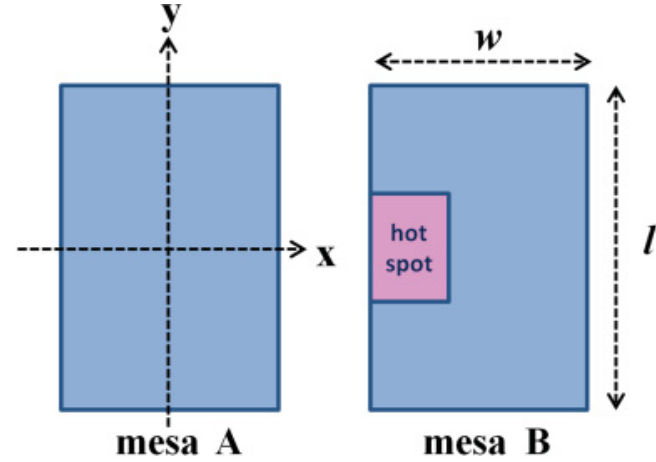

mesa B
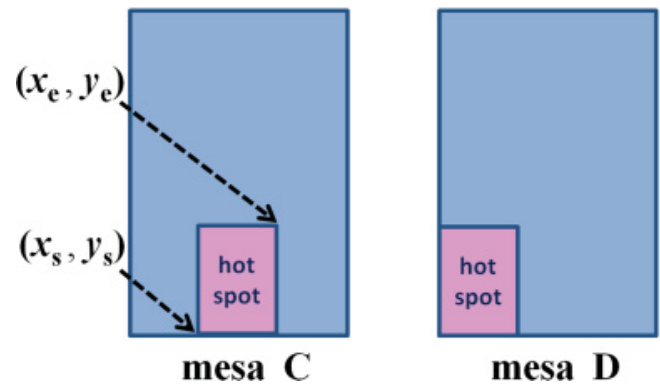

FIG. 2. (Color online) Schematic figures of the positions of the hot spots in the four different mesas.

the mesa. The far-field radiation patterns are calculated from the equivalent electric and magnetic current along the surface of the calculation region. ${ }^{23}$

For investigating the effect of an inhomogeneity on the radiation properties, we consider the appearance of "hot spots" where the temperature is locally high. In our model, such hot spots are simulated by decreasing the local critical current. We consider four types of mesas (mesas AD). Mesa A does not have hot spots, and mesas B-D have one hot spot each. We take $j_{c}^{\prime}(x, y)=1-\gamma$ in the hot-spot region $\left(x_{s}<x<x_{e}, y_{s}<y<y_{e}\right)$ and $j_{c}^{\prime}(x, y)=$ 1 in the other region. The center of the mesa is located at the origin, and we choose $x_{s}=-0.24 \lambda_{c}, x_{e}=$ $-0.08 \lambda_{c}, y_{s}=-0.12 \lambda_{c}, y_{e}=0.12 \lambda_{c}$ for mesa $\mathrm{B}, x_{s}=$ $-0.08 \lambda_{c}, x_{e}=0.08 \lambda_{c}, y_{s}=-0.36 \lambda_{c}, y_{e}=-0.12 \lambda_{c}$ for mesa $\mathrm{C}$, and $x_{s}=-0.24 \lambda_{c}, x_{e}=-0.08 \lambda_{c}, y_{s}=-0.36 \lambda_{c}, y_{e}=$ $-0.12 \lambda_{c}$ for mesa D. Figure 2 shows schematic figures of the geometries of these four mesas. The appearance of such hot spots whose positions are asymmetric with respect to the center of the mesas have been already reported in previous experimental studies. ${ }^{20-22}$

\section{RESULTS AND DISCUSSIONS}

First, we examine the radiation power versus voltage $(P-V)$ curves of the mesas. The radiation powers are calculated from the time average of the surface integration of the Poynting vector given by $(c / 4 \pi) \int_{S}(\boldsymbol{E} \times \boldsymbol{H}) \cdot \boldsymbol{n} d S$, where $S$ indicates the outer boundary of the calculation region and $\boldsymbol{n}$ is the unit vector normal to the boundary. Figures 3(a)-3(d) show the $P-V$ curves of mesas A-D, respectively, for $\gamma=0.1-0.3$. Here the power and the voltage are normalized by $P_{0}=$ $c \Phi_{0}^{2} / 16 \pi d^{3}$ and $V_{p}=\hbar \omega_{p} / 2 e$, respectively. With respect to 
(a)

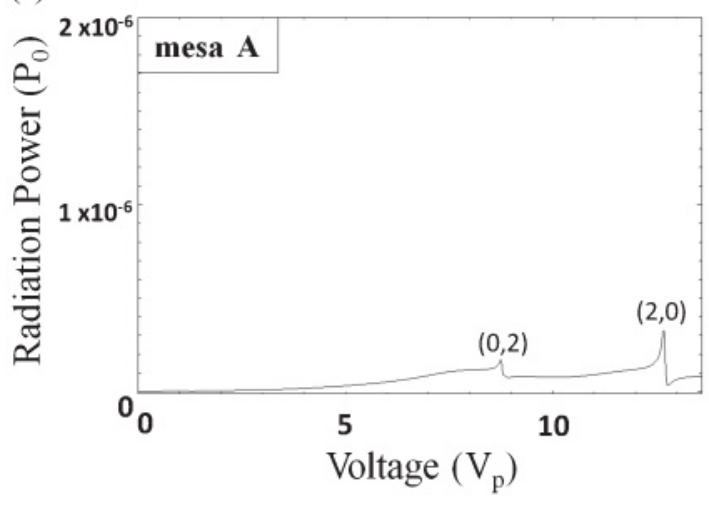

(c)

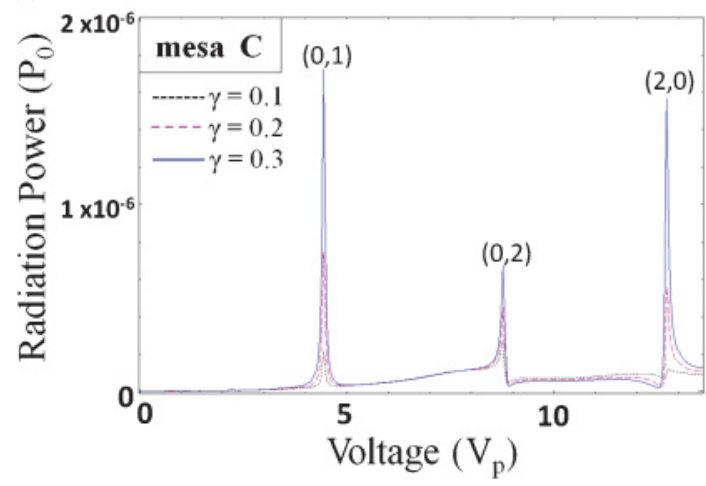

(b)

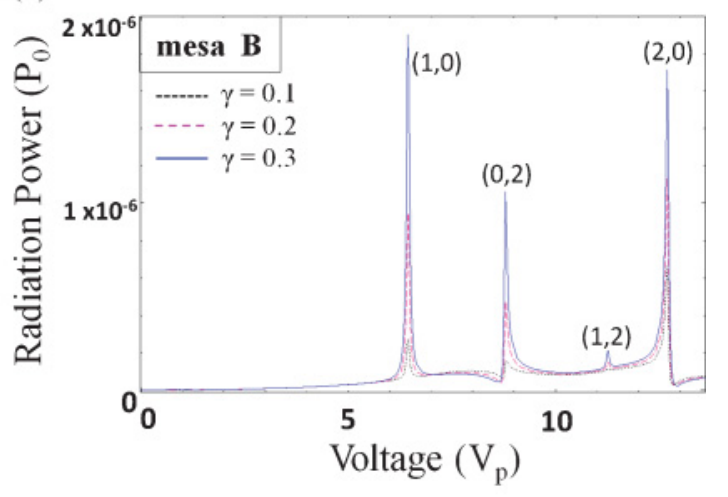

(d)

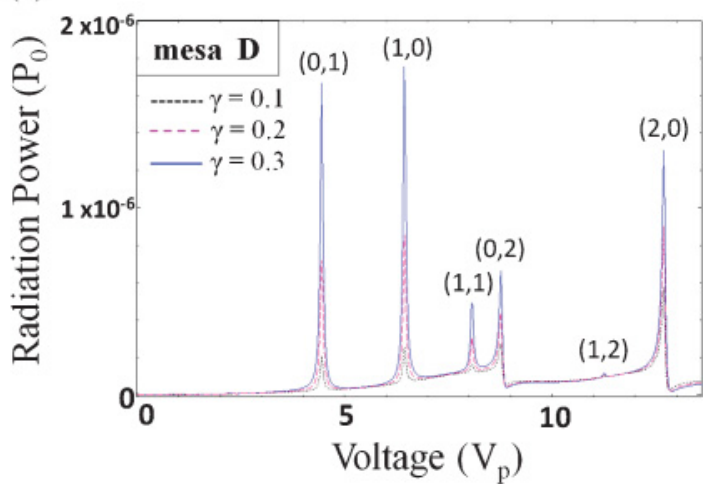

FIG. 3. (Color online) (a) The $P-V$ curve of the homogeneous mesa. (b)-(d) The $P-V$ curves of the inhomogeneous mesas with hot spots. The black (dotted), red (broken) and blue (solid) lines indicate the curves for $\gamma=0.1, \gamma=0.2$, and $\gamma=0.3$ respectively.

mesas B-D, we have calculated $P-V$ curves for $\gamma=0.1-0.3$. In Figs. 3(a)-3(d), we can see sharp peaks at voltages where the ac Josephson frequency $f_{\mathrm{J}}=2 e V / h$ satisfies the cavity resonance condition $f_{\mathbf{J}}=c \sqrt{(m / 2 w)^{2}+(n / 2 l)^{2}} / \sqrt{\epsilon_{c}}$, where $m$ and $n$ are arbitrary integers. The peak voltage $V=6.44 V_{p}$, for example, satisfies $(m, n)=(1,0)$. The labels of the peaks in Fig. 3 indicate the indices of the cavity resonance modes. It should be noted that the peak voltages slightly deviate from the voltages that are estimated from the above cavity resonance condition due to the fringing effect. If we take $d=1.2 \times 10^{-7} \mathrm{~cm}$, the peak powers become $\sim 10 \mu \mathrm{W}$. These values are comparable to those reported in experimental works. ${ }^{1-3}$ As can be seen from Fig. 3, the cavity resonant peaks whose $m$ and $n$ are even appear in all mesas. Meanwhile, the peaks whose $m$ is odd only appear when the $y$-axis symmetry is broken (mesas B and D), and the peaks whose $n$ is odd only appear when the $x$-axis symmetry is broken (mesas $\mathrm{C}$ and $\mathrm{D}$ )

In the mesa-structured IJJs, the excitation of the electromagnetic mode comes from the ac Josephson current flowing through the mesa, and the distribution of the ac Josephson current has a symmetry corresponding to the mesa structure unless the solitonic states appear. Hence, in a homogeneous rectangular mesa such as mesa $\mathrm{A}$, the odd-numbered cavity modes whose electric fields are asymmetric with respect to the center of the mesa cannot be excited. Meanwhile, in mesas B-D, the odd-numbered cavity modes appear because the hot spots break the reflectional symmetries of the rectangular mesas. Furthermore, the increase of the asymmetric nature of the mesa enhances the mode excitation, as can be seen from the results for $\gamma=0.1-0.3$ in Figs. 3(b) $-3(d)$.

Next, we investigate the internal mode of the mesa at the peak voltages. Hereafter, we show the results of mesa D for $\gamma=0.2$ because all cavity modes appear in this mesa. To investigate the oscillation part of the electric field, we take the Fourier transform of the electric fields $E_{z}$ in the mesa. Figures 4(a)-4(f) show the amplitude maps of the Fourier components of $E_{z}$ at the ac Josephson frequency for $V=4.47 V_{p}, V=6.44 V_{p}, V=8.07 V_{p}, V=8.77 V_{p}, V=$ $11.3 V_{p}$, and $V=12.7 V_{p}$, respectively. Figure 4 shows the appearance of the standing waves corresponding to the cavity resonance modes. However, the amplitudes of the electric fields at the positions of the nodes have finite values. Since the shapes of the standing waves are clearly seen in Fig. 4, this fact indicates the existence of background oscillating modes that are almost uniform in the mesas. If we assume the uniform background modes, the oscillation part of the electric field $E_{\mathrm{OS}}$ in the mesa can be written as

$$
\begin{aligned}
E_{\mathrm{OS}}(x, y, t)= & E_{\mathrm{bg}} \sin \left(2 \pi f_{c} t\right)+E_{\mathrm{cv}} \cos \left(\frac{m \pi x}{w}+\frac{m \pi}{2}\right) \\
& \times \cos \left(\frac{n \pi y}{l}+\frac{n \pi}{2}\right) \sin \left(2 \pi f_{c} t+\varphi_{0}\right) \\
& (-w / 2<x<w / 2,-l / 2<y<l / 2),
\end{aligned}
$$




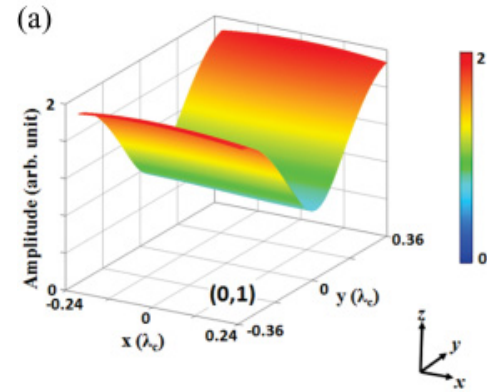

(d)

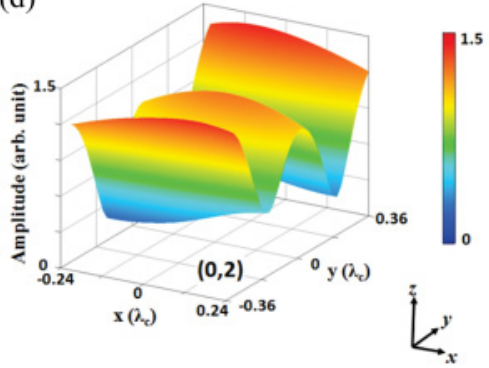

(b)

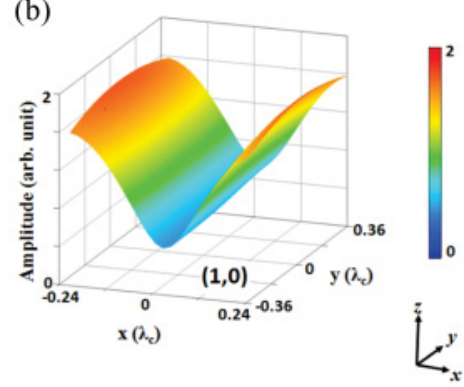

(e)

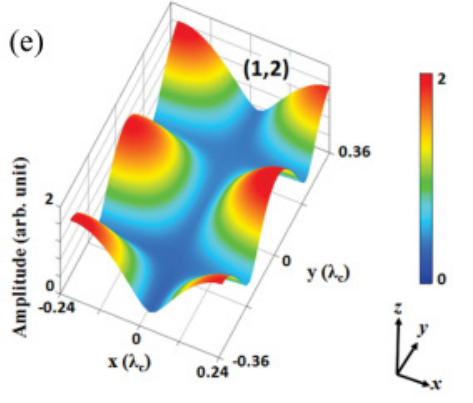

(c)

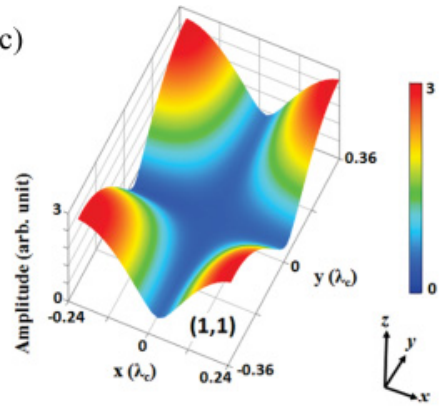

(f)

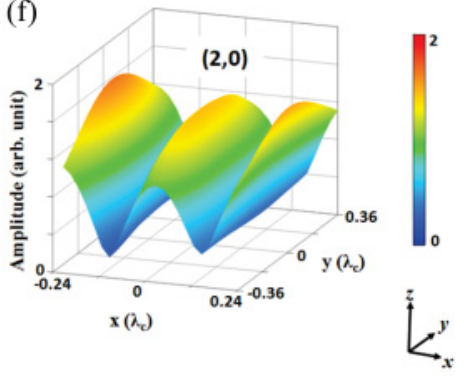

FIG. 4. (Color online) The Fourier components of electric fields $E_{z}$ at (a) $V=4.47 V_{p}$, (b) $V=6.44 V_{p}$, (c) $V=8.07 V_{p}$, (d) $V=8.77 V_{p}$, (e) $V=11.3 V_{p}$, and (f) $V=12.7 V_{p}$.

where $E_{\mathrm{bg}}$ and $E_{\mathrm{cv}}$ are, respectively, the amplitudes of the uniform background mode and the cavity resonance mode, $f_{c}$ is the cavity resonance frequency $c \sqrt{(m / 2 w)^{2}+(n / 2 l)^{2}} / \sqrt{\epsilon_{c}}$, and $\varphi_{0}$ is the phase difference between these modes. This kind of "dual" internal modes has already been proposed in a previous theoretical study. ${ }^{17}$ However, that study did not calculate the phase dynamics in the IJJs and did not confirm the existence of this mode by numerical calculations.

Here we examine the results at $V=6.44 V_{p}$ which satisfy the $(1,0)$ cavity resonance condition to see how well this dual-mode model [Eq. (3)] can describe the numerical results.

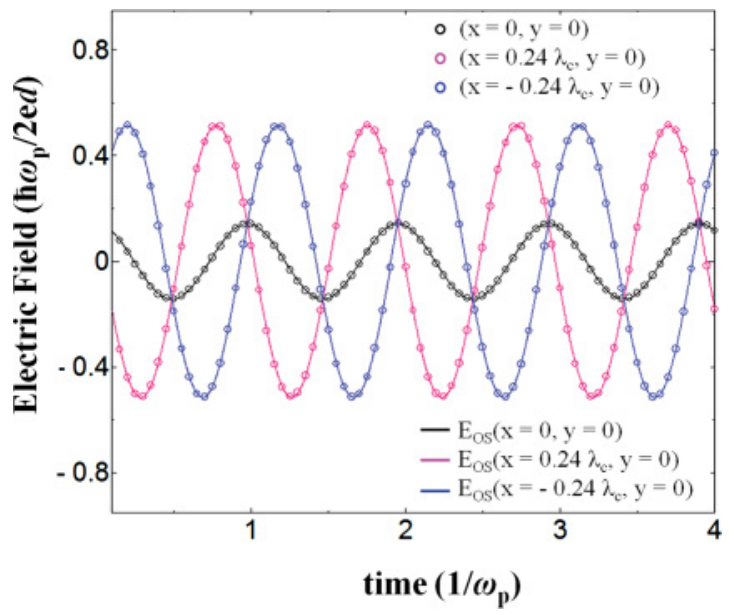

FIG. 5. (Color online) The time evolution of the oscillation part of electric fields at $V=6.44 V_{p}$. The black, red, and blue open circles indicate the calculated values in our simulation at $(x, y)=(0,0)$, $(x, y)=\left(0.24 \lambda_{c}, 0\right)$, and $(x, y)=\left(-0.24 \lambda_{c}, 0\right)$, respectively. The lines indicate $E_{\mathrm{OS}}$ at each position when $m=1, n=0, E_{\mathrm{bg}}=0.142$, $E_{\mathrm{cv}}=0.492$, and $\varphi_{0}=0.5 \pi$.
Figure 5 shows the time evolution of the oscillation part of electric fields at three positions in the mesa: $(x, y)=$ $(0,0),(x, y)=\left(0.24 \lambda_{c}, 0\right)$, and $(x, y)=\left(-0.24 \lambda_{c}, 0\right)$. The open circles indicate the calculated values in our simulation using Eqs. (1) and (2), and the lines indicate $E_{\mathrm{OS}}(x, y, t)$ obtained from Eq. (3) when $m=1, n=0, E_{\mathrm{bg}}=0.142, E_{\mathrm{cv}}=0.492$, and $\varphi_{0}=0.5 \pi$. As seen in Fig. 5, the calculated values are well described by Eq. (3). Moreover, the amplitude distribution of $E_{\mathrm{OS}}$ becomes $\sim \sqrt{E_{\mathrm{bg}}^{2}+E_{\mathrm{cv}}^{2} \sin (\pi x / w)^{2}}$ when the phase difference $\varphi_{0} \sim 0.5 \pi$, and this distribution agrees well with the amplitude distribution at $V=6.44 V_{p}$, shown in Fig. 4(b). Next, we examine the tail region of the resonance peak. Although $\varphi_{0}$ is close to $0.5 \pi$ at the peak voltage, $\varphi_{0}$ deviates from $0.5 \pi$ in the tail region of the peak. Figure 6(a) shows the time evolution of the oscillation part of electric fields at $V=6.37 V_{p}$. The open circles indicate the calculated values in our simulation, and the lines indicate $E_{\mathrm{OS}}(x, y, t)$ obtained from Eq. (3) when $m=1, n=$ $0, E_{\mathrm{bg}}=0.144, E_{\mathrm{cv}}=0.244$, and $\varphi_{0}=0.831 \pi$. Again, the simulated data are described very well by Eq. (3). Figure 6(b) shows the amplitude maps of the Fourier components of $E_{z}$ at the ac Josephson frequency for $V=6.37 V_{p}$. Since $\varphi_{0}$ deviates from $0.5 \pi$ in this voltage region, the amplitude distribution of $E_{\mathrm{OS}}$ becomes asymmetric and is given by $\sqrt{E_{\mathrm{bg}}^{2}+E_{\mathrm{cv}}^{2} \sin (\pi x / w)^{2}+2 E_{\mathrm{bg}} E_{\mathrm{cv}} \sin (\pi x / w) \cos \left(\varphi_{0}\right)}$. This agrees well with the simulated amplitude map of $E_{z}$ at the ac Josephson frequency for $V=6.37 V_{p}$, shown in Fig. 6(b).

Furthermore, to clarify the relation between the internal modes and radiation patterns, we calculate the threedimensional radiation patterns at peak voltages. Figure 7(a) shows three-dimensional plots of the radiation intensity $I(\theta, \phi)$ at $V=6.44 V_{p}$, and Fig. 7(b) shows the polar plots of $I\left(\theta, 90^{\circ}\right)$ in the $x-z$ plane and $I\left(\theta, 0^{\circ}\right)$ in the $y-z$ plane at $V=6.44 V_{p}$. We can see from Fig. 7 that the radiation pattern shows strong 
(a)

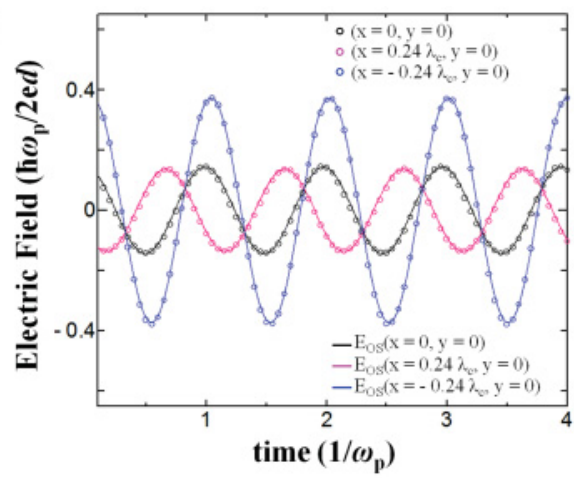

(b)

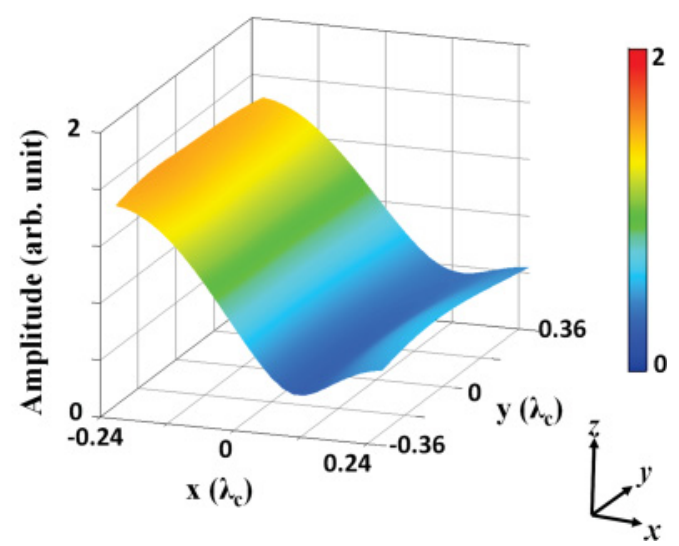

FIG. 6. (Color online) (a) The time evolution of the oscillation part of electric fields at $V=6.37 V_{p}$. The black, red, and blue open circles indicate the calculated values in our simulation at $(x, y)=$ $(0,0),(x, y)=\left(0.24 \lambda_{c}, 0\right)$, and $(x, y)=\left(-0.24 \lambda_{c}, 0\right)$, respectively. The lines indicate $E_{\mathrm{OS}}$ at each position when $m=1, n=0, E_{\mathrm{bg}}=$ $0.144, E_{\mathrm{cv}}=0.244$, and $\varphi_{0}=0.831 \pi$. (b) The amplitude maps of the Fourier components of $E_{z}$ at ac Josephson frequency for $V=6.37 V_{p}$.

asymmetry with respect to the $y-z$ plane and is different from that of the usual patch antenna satisfying the $(1,0)$ cavity resonance condition. This asymmetry is explained by the interference between electromagnetic waves emitted by two different radiation sources. As previously mentioned, the internal modes of the mesas are described by the sum of the background modes and cavity resonance modes. Hence, the total electromagnetic wave is also described by the sum of the electromagnetic waves emitted by these modes. If we assume a uniform background mode similar to the above discussion, the background mode emits the electromagnetic wave whose electric field is symmetric with respect to the $y-z$ plane. On the other hand, the $(1,0)$ cavity resonance mode emits an electromagnetic wave whose electric field is antisymmetric with respect to the $y-z$ plane. Therefore, radiation intensity becomes strong by constructive interference on one side of the $y-z$ plane and weak by destructive interference on the opposite side because of the different symmetries of the radiation waves.

In Figs. 8(a) and 8(b), we show $I(\theta, \phi), I\left(\theta, 90^{\circ}\right)$, and $I\left(\theta, 0^{\circ}\right)$ at $V=12.7 V_{p}$, which satisfies the $(2,0)$ cavity resonance condition. The radiation intensity decreases to zero at $\theta=0^{\circ}$, similar to that arising from dipole antenna radiation. In contrast to the result obtained from the $(1,0)$ cavity resonance condition, the radiation pattern is almost symmetric with respect to the $y-z$ plane when the voltage satisfies the $(2,0)$ cavity resonance condition. This is because the $(2,0)$ cavity mode emits an electromagnetic wave whose electric field is symmetric with respect to the $y-z$ plane, similar to the uniform background mode. In this case, interference between the electromagnetic waves emitted by both modes results in similar radiation intensities on each side of the $y-z$ plane. The small asymmetric nature shown in Figs. 8(a) and 8 (b) reflects the fact that the background mode is slightly asymmetric because of the $j_{c}$ modulation at the hot spot. It is noteworthy that the radiation intensity in the $y$ direction, which is normal to the wave vector of the standing wave, is small but finite, as shown in Fig. 8(b). The usual patch antenna satisfying the $(2,0)$ cavity modes does not emit the electromagnetic wave in this direction. Thus, the radiation in this direction indicates the existence of the uniform background mode.

In Figs. 9(a)-9(d), we show $I(\theta, \phi)$ at $V=4.47 V_{p}$, $V=8.07 V_{p}, V=8.77 V_{p}$, and $V=11.3 V_{p}$. Similar to the radiation pattern at $V=6.44 V_{p}$, the large asymmetric nature shown in Figs. 9(a), 9(b), and 9(d) comes from the interference between the electromagnetic waves emitted by the cavity resonance mode and the uniform background mode. It is important to keep in mind that the diffraction at substrate
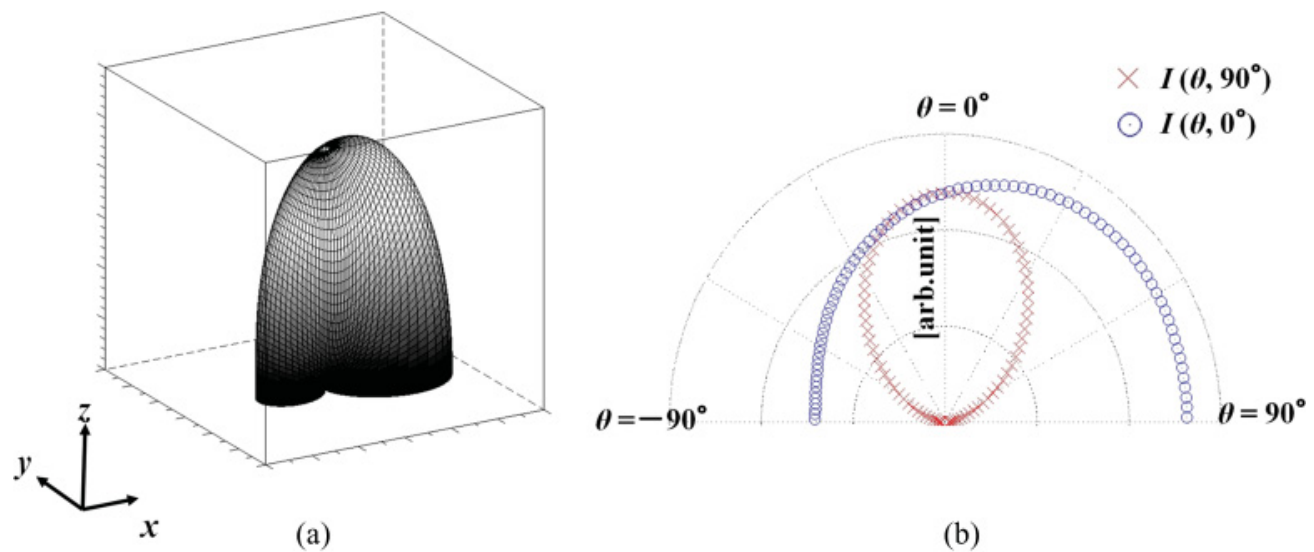

FIG. 7. (Color online) (a) The three-dimensional plots of $I(\theta, \phi)$ at $V=6.44 V_{p}$. (b) The polar plots of $I\left(\theta, 0^{\circ}\right)$ in the $x-z$ plane and $I\left(\theta, 90^{\circ}\right)$ in the $y-z$ plane at $V=6.44 V_{p}$. 


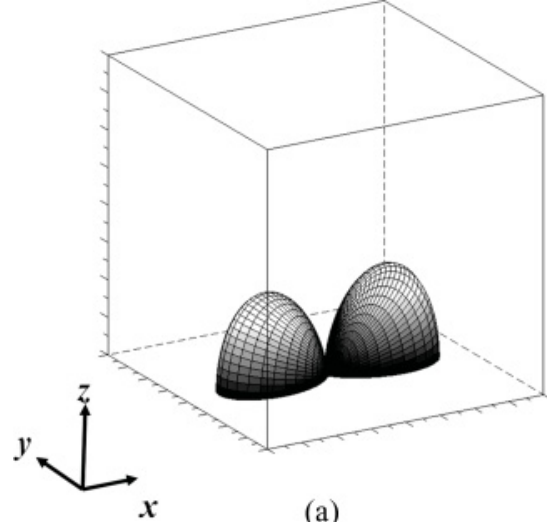

(a)

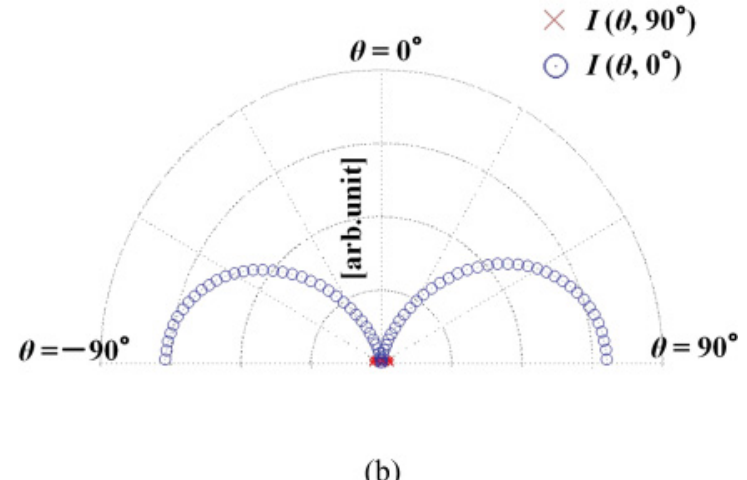

(b)

FIG. 8. (Color online) (a) The three-dimensional plots of $I(\theta, \phi)$ at $V=12.7 V_{p}$. (b) The polar plots of $I\left(\theta, 0^{\circ}\right)$ in the $x-z$ plane and $I\left(\theta, 90^{\circ}\right)$ in the $y-z$ plane at $V=12.7 V_{p}$.

edges also affects the total radiation pattern. In particular, the radiation patterns have been measured experimentally for mesas whose substrate sizes are comparable to the wavelength of the radiation waves. In this case, the radiation patterns greatly change from those in which the mesas sat upon an infinite ground plane. ${ }^{24,25}$ Therefore, for the analysis of the experimental radiation patterns, we need to take into account the effect of the diffraction at the substrate edges whose geometries are similar to the experimental setups. ${ }^{26}$

Finally, we would like to discuss the $P-V$ characteristic from the view point of Fano resonance. ${ }^{27}$ As shown in Figs. 3(a)-3(d), the peaks corresponding to the even-numbered cavity resonance mode show asymmetry Fano resonance peaks. Fano resonances appear in systems where continuum and discrete states coexist, such as the quantum dot in an Aharonov-Bohm ring, ${ }^{28}$ and the asymmetric nature of physical quantities with respect to the energy comes from the interference between these continuum and discrete states. In the in-phase IJJs under dc bias voltages, the Josephson plasma mode that uniformly oscillates inside the IJJs appears for any voltage like the continuum mode, and this mode corresponds to the uniform background mode discussed above. Meanwhile, similar to the discrete mode, the Josephson plasma modes oscillating with electromagnetic standing waves appear only
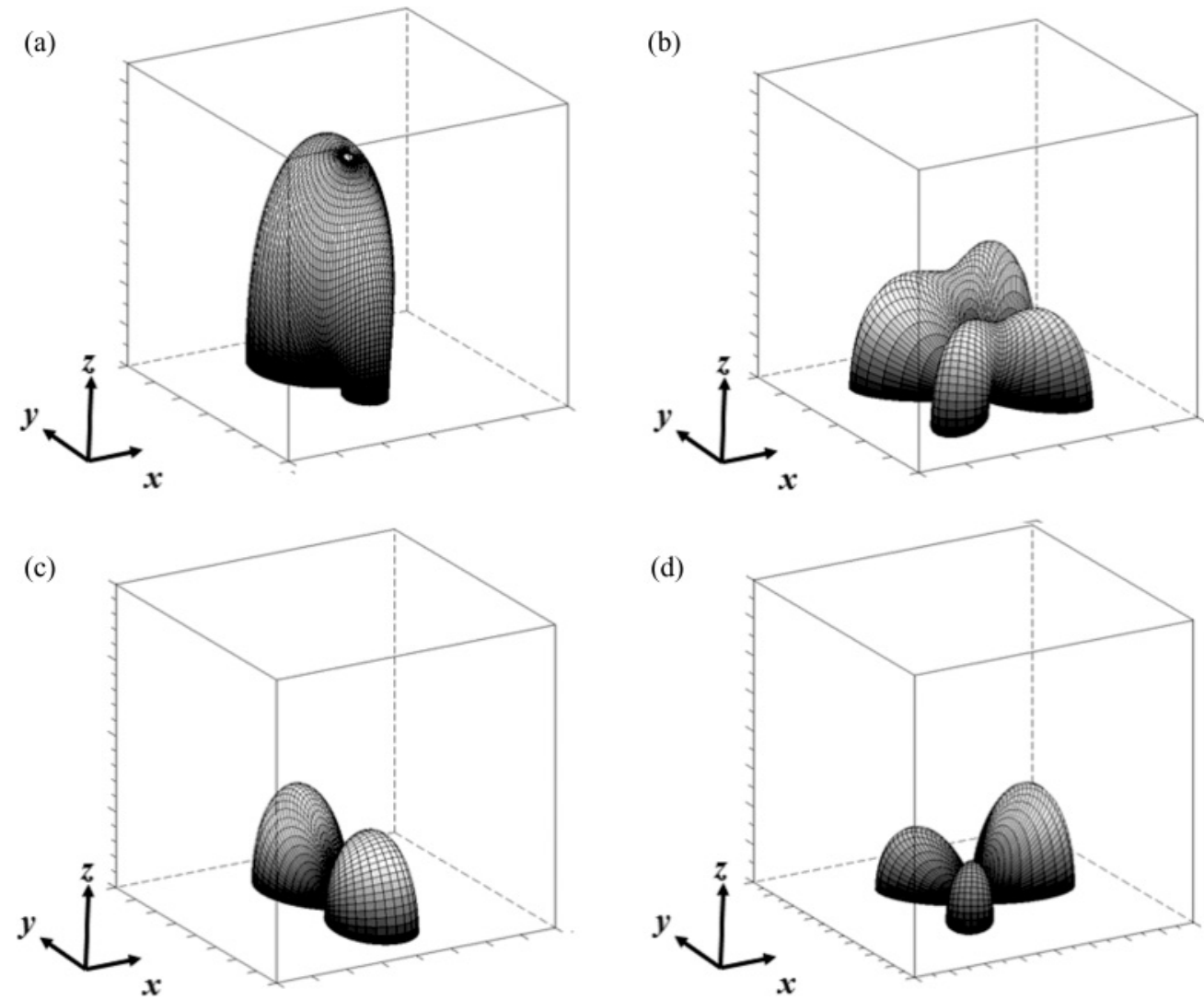

FIG. 9. The three-dimensional plots of $I(\theta, \phi)$ at (a) $V=4.47 V_{p}$, (b) $V=8.07 V_{p}$, (c) $V=8.77 V_{p}$, (d) and $V=11.3 V_{p}$. 
when the voltage satisfies the cavity resonance condition. Since the radiation power is related to the strength of the plasma mode excitation inside the IJJs, the asymmetric peaks in $P-V$ curves can be regarded as Fano resonance peaks coming from the interference between the continuum and discrete Josephson plasma modes. It should be noted that this asymmetric peak does not appear for the odd-numbered cavity resonance condition. In the case of the odd-numbered cavity resonance condition, the discrete Josephson plasma modes become antisymmetric with respect to the center of the mesa, while the continuum modes are symmetric. Thus, the interference between the continuum and discrete plasma modes becomes very weak, and the Fano resonance peak disappears. The precise measurement of the line shape of the peaks in the $P-V$ curves is desired to confirm the resonance between these two modes.

\section{CONCLUSION}

In this study, we have studied numerically the threedimensional radiation patterns from mesa-structured intrinsic Josephson junctions. We assumed an in-phase mode of the phase differences and calculated electromagnetic fields inside and outside of IJJs simultaneously. We considered the appearance of hot spots in the mesa where $j_{c}$ locally decreases and investigated the change of the radiation power with the positions of the hot spots. We observe strong radiation when the ac Josephson frequency satisfies the cavity resonance condition. The transverse-magnetic modes $\mathrm{TM}_{m, n}$ whose indices $m$ and $n$ are even appear regardless of the positions of hot spots. On the other hand, $\mathrm{TM}_{m, n}$ cavity modes whose $m$ or $n$ are odd appear only when the hot spots break the reflectional symmetry of the mesa structure. The odd-numbered cavity modes are exited by the ac Josephson currents whose distributions are asymmetric with respect to the center of the mesa. Moreover, the radiation patterns reflect the coexistence of two types of internal modes, that is, a uniform background mode and a cavity resonance mode. In particular, the radiation patterns for odd-numbered cavity resonance conditions exhibit asymmetry because of the interference between these two types of radiation waves. Furthermore, in the case of even-numbered cavity resonance conditions, the interference between these two types of Josephson plasma modes induces asymmetric Fano-like peaks in the $P-V$ curves.

In this study, hot spots are simulated by decreasing the local $j_{c}$. Several theoretical studies have also investigated the radiation properties in rectangular mesas having an asymmetric $j_{c}$ modulation using a two-dimensional calculation model; ${ }^{11,15}$ however, they did not mention the origin of the $j_{c}$ modulation. Furthermore, the change of internal modes by modulations in the $a b$ plane and three-dimensional radiation patterns emitted by these modes were not clarified in these two-dimensional analyses. As reported in this paper, the appearance of hot spots strongly affects the radiation power. Therefore, the control of hot spots will be one of the key issues for obtaining stable and strong radiation from IJJs.

\section{ACKNOWLEDGMENTS}

We wish to thank R. A. Klemm (University of Central Florida) and S. Fukuya for valuable discussions. This work was supported in part by CREST-JST (Japan Science and Technology Agency). *hide@ims.tsukuba.ac.jp

${ }^{1}$ L. Ozyuzer, A. E. Koshelev, C. Kurter, N. Gopalsami, Q. Li, M. Tachiki, K. Kadowaki, T. Yamamoto, H. Minami, H. Yamaguchi, T. Tachiki, K. E. Gray, W.-K. Kwok, and U. Welp, Science 318, 1291 (2007).

${ }^{2}$ H. Minami, I. Kakeya, H. Yamaguchi, T. Yamamoto, and K. Kadowaki, Appl. Phys. Lett. 95, 232511 (2009).

${ }^{3}$ K. Kadowaki, M. Tsujimoto, K. Yamaki, T. Yamamoto, T. Kashiwagi, H. Minami, M. Tachiki, and R. Klemm, J. Phys. Soc. Jpn. 79, 023703 (2010).

${ }^{4}$ M. Tsujimoto, K. Yamaki, K. Deguchi, T. Yamamoto, T. Kashiwagi, H. Minami, M. Tachiki, K. Kadowaki, and R. A. Klemm, Phys. Rev. Lett. 105, 037005 (2010).

${ }^{5}$ H. Minami, N. Orita, T. Koike, T. Yamamoto, and K. Kadowaki, Phys. C 470, S822 (2010).

${ }^{6}$ T. Kashiwagi, K. Yamaki, M. Tsujimoto, K. Deguchi, N. Orita, T. Koike, R. Nakayama, H. Minami, T. Yamamoto, R. A. Klemm, M. Tachiki, and K. Kadowaki, J. Phys. Soc. Jpn. 80, 094709 (2011). ${ }^{7}$ T. M. Benseman, A. E. Koshelev, K. E. Gray, W.-K. Kwok, U. Welp, K. Kadowaki, M. Tachiki, and T. Yamamoto, Phys. Rev. B 84, 064523 (2011).

${ }^{8}$ T. Kashiwagi, M. Tsujimoto, T. Yamamoto, H. Minami, K. Yamaki, K. Delfanazari, K. Deguchi, N. Orita, T. Koike, R. Nakayama, T. Kitamura, M. Sawamura, S. Hagino, K. Ishida, K. Ivanovic, H. Asai, M. Tachiki, R. A. Klemm, and K. Kadowaki, Jpn. J. Appl. Phys. (to be published 2012).
${ }^{9}$ S. Lin and X. Hu, Phys. Rev. Lett. 100, 247006 (2008).

${ }^{10}$ X. Hu and S. Lin, Phys. Rev. B 78, 134510 (2008).

${ }^{11}$ A. E. Koshelev and L. N. Bulaevskii, Phys. Rev. B 77, 014530 (2008).

${ }^{12}$ A. E. Koshelev, Phys. Rev. B 78, 174509 (2008).

${ }^{13}$ H. Matsumoto, T. Koyama, and M. Machida, Phys. C 469, 1600 (2009).

${ }^{14}$ T. Koyama, H. Matsumoto, M. Machida, and K. Kadowaki, Phys. Rev. B 79, 104522 (2009).

${ }^{15}$ T. Koyama, H. Matsumoto, M. Machida, and Y. Ota, Supercond. Sci. Technol. 24, 085007 (2011).

${ }^{16}$ M. Tachiki, S. Fukuya, and T. Koyama, Phys. Rev. Lett. 102, 127002 (2009).

${ }^{17}$ R. A. Klemm and K. Kadowaki, J. Supercond. Novel Magn. 23, 613 (2010).

${ }^{18}$ R. A. Klemm and K. Kadowaki, J. Phys. Condens. Matter 22, 375701 (2010).

${ }^{19}$ R. A. Klemm, E. R. LaBerge, D. R. Morley, T. Kashiwagi, M. Tsujimoto, and K. Kadowaki, J. Phys. Condens. Matter 23, 025701 (2011).

${ }^{20}$ H. B. Wang, S. Guenon, J. Yuan, A. Iishi, S. Arisawa, T. Hatano, T. Yamashita, D. Koelle, and R. Kleiner, Phys. Rev. Lett. 102, 017006 (2009).

${ }^{21}$ H. B. Wang, S. Guenon, B. Gross, J. Yuan, Z. G. Jiang, Y. Y. Zhong, M. Gruenzweig, A. Iishi, P. H. Wu, T. Hatano, D. Koelle, and R. Kleiner, Phys. Rev. Lett. 105, 057002 (2010). 
${ }^{22}$ S. Guenon, M. Grunzweig, B. Gross, J. Yuan, Z. G. Jiang, Y. Y. Zhong, M. Y. Li, A. Iishi, P. H. Wu, T. Hatano, R. G. Mints, E. Goldobin, D. Koelle, H. B. Wang, and R. Kleiner, Phys. Rev. B 82, 214506 (2010).

${ }^{23}$ R. J. Luebbers, K. S. Kunz, M. Schneider, and F. Hunsberger, IEEE Trans. Antennas Propag. 39, 429 (1991).

${ }^{24}$ C. A. Balanis, Antenna Theory: Analysis and Design, 3rd ed. (Wiley, Hoboken, NJ, 2005).
${ }^{25}$ J. Huang, IEEE Trans. Antennas Propag. 31, 649 (1983).

${ }^{26}$ H. Asai, M. Tachiki, T. Kashiwagi, H. Minami, T. Yamamoto, and K. Kadowaki, in Proceedings of the 26th International Conference on Low Temperature Physics, Beijing, 2012 (to be published in J. Phys. Conf. Ser.).

${ }^{27}$ U. Fano, Phys. Rev. 124, 1866 (1961).

${ }^{28}$ K. Kobayashi, H. Aikawa, S. Katsumoto, and Y. Iye, Phys. Rev. Lett. 88, 256806 (2002). 